Bull. Korean Math. Soc. 50 (2013), No. 6, pp. 1957-1972

http://dx.doi.org/10.4134/BKMS.2013.50.6.1957

\title{
REFLEXIVE PROPERTY ON IDEMPOTENTS
}

\author{
Tai Keun Kwak and Yang Lee
}

\begin{abstract}
The reflexive property for ideals was introduced by Mason and has important roles in noncommutative ring theory. In this note we study the structure of idempotents satisfying the reflexive property and introduce reflexive-idempotents-property (simply, RIP) as a generalization. It is proved that the RIP can go up to polynomial rings, power series rings, and Dorroh extensions. The structure of non-Abelian RIP rings of minimal order (with or without identity) is completely investigated.
\end{abstract}

\section{Introduction}

Throughout this paper all rings are associative with identity unless otherwise stated. Given a ring $R$ the polynomial ring (resp., the power series ring) with an indeterminate $x$ over $R$ is denoted by $R[x]$ (resp., $R[[x]]$ ). For any polynomial $f(x)$ in $R[x]$, let $C_{f(x)}$ denote the set of all coefficients of $f(x)$. Denote the $n$ by $n$ full matrix ring over $R$ by $\operatorname{Mat}_{n}(R)$ and the $n$ by $n$ upper triangular matrix ring over $R$ by $U_{n}(R)$. Use $E_{i j}$ for the matrix with $(i, j)$-entry 1 and elsewhere 0. Let $I d(R)$ be the set of all idempotent elements of $R$. Denote $\left\{a \in U_{n}(R) \mid\right.$ the diagonal entries of $a$ are all equal $\}$ by $D_{n}(R)$. $\mathbb{Z}$ and $\mathbb{Z}_{n}$ denote the ring of integers and the ring of integers modulo $n$, respectively. $G F\left(p^{n}\right)$ denotes the Galois field of order $p^{n}$ for a prime $p$ and $n \geq 1$. J $(R)$ denotes the Jacobson radical of $R .|S|$ denotes the cardinality of given a set $S$.

Mason [18] introduced the reflexive property for ideals, and then this concept was generalized by Kim and Baik [9, 10] by defining idempotent reflexive right ideals and rings. A right ideal $I$ of a ring $R$ (possibly without identity) is called reflexive [18] if $a R b \subseteq I$ implies $b R a \subseteq I$ for $a, b \in R$. $R$ is called reflexive if 0 is a reflexive ideal (i.e., $a R b=0$ implies $b R a=0$ for $a, b \in R$.) In [12], Kwak and Lee characterized aspects of the reflexive and one-sided idempotent reflexive properties, showing that the concept of idempotent reflexive ring is not leftright symmetric [12, Example 3.3]. For a one-sided ideal $I$ of a $\operatorname{ring} R, I$ is

Received July 19, 2012; Revised March 21, 2013.

2010 Mathematics Subject Classification. 16S99, $16 \mathrm{U} 80$.

Key words and phrases. reflexive property, reflexive-idempotents-property (RIP), polynomial ring, Dorroh extension, minimal RIP ring. 
called right idempotent reflexive [12, Definition 3.1] if aRe $\subseteq I$ implies $e R a \subseteq I$ for any $a, e^{2}=e \in R$, and a ring $R$ is called right idempotent reflexive if 0 is a right reflexive ideal. Left idempotent reflexive ideals and rings are defined similarly. If a ring is both left and right idempotent reflexive, then the ring is called an idempotent reflexive ring (refs. [9, 10]). Reflexive rings are obviously one-sided idempotent reflexive, but not conversely by [12, Example 2.3(1)]. It is proved that the reflexive condition is Morita invariant [12, Theorem 2.6]. A (right idempotent) reflexive ring which is not semiprime (resp., reflexive) is also constructed from any semiprime (resp., reflexive) ring [12, Proposition 2.5 and Theorem 3.9].

Recall that a ring is reduced if it has no nonzero nilpotent elements. Cohn [4] called a ring $R$ reversible if $a b=0$ implies $b a=0$ for $a, b \in R$. Due to Bell [2], a right (or left) ideal $\mathrm{I}$ of a ring $\mathrm{R}$ is said to have the insertion of factors property (simply, IFP) if $a b \in I$ implies $a R b \subseteq I$ for $a, b \in R$. A ring $R$ is called IFP if the zero ideal of $R$ has the IFP. A ring $R$ is called 2-primal [3] if the prime radical of $R$ coincides with the set of all nilpotent elements of $R$. In [17], a ring $R$ is called $N I$ if the upper nilradical of $R$ coincides with the set of all nilpotent elements of $R$. IFP rings are 2-primal and 2-primal rings are NI, but the converses are not true. Also note that the prime radical of a 2-primal ring and the upper nilradical of an NI ring are reflexive ideals.

Rege and Chhawchharia [20] called a ring $R$ Armendariz if $a b=0$ for all $a \in C_{f(x)}$ and $b \in C_{g(x)}$ whenever any polynomials $f(x), g(x)$ over $R$ satisfy $f(x) g(x)=0$. A ring is called Abelian if every idempotent in it is central. It is well-known that IFP rings and Armendariz rings are Abelian. Every Abelian ring is idempotent reflexive, and hence IFP rings and Armendariz rings are idempotent reflexive. Note that reversible rings are reflexive by $[12$, Proposition 2.2], but IFP and the reflexive ring property don't imply each other by [12, Example 2.3].

In this paper, we study on rings with reflexive-idempotents-property (simply, RIP) which is a generalization of one-sided idempotent reflexive rings. It is proved that the one-sided idempotent reflexive property and the reflexiveidempotents-property coincide for a right principally quasi-Baer ring (Proposition 2.11), and that $R$ is an RIP ring if and only if $R[x]$ is an RIP ring if and only if $R[[x]]$ is an RIP ring (Theorem 3.1), and that a ring $R$ is RIP if and only if $D_{n}(R)$ is RIP (Theorem 3.3). If $R$ is a minimal non-Abelian RIP ring, then $R$ is of order 16 and is isomorphic to $\operatorname{Mat}_{2}\left(\mathbb{Z}_{2}\right)$ (Theorem 4.2). We additionally give an example that RIP rings need not be directly finite (Example 2.13).

\section{Properties of RIP rings}

We begin with the following.

Definition 2.1. A ring $R$ is called to have the reflexive-idempotents-property (simply, RIP) if $R$ satisfies the property that

$$
e R f=0 \text { implies } f R e=0 \text { for any } e, f \in I d(R) .
$$


A ring shall be called $R I P$ if it satisfies the reflexive-idempotents-property.

It can be easily checked that every one-sided idempotent reflexive ring is RIP, entailing that Abelian rings are RIP. Hence, the class of RIP rings contains IFP rings and Armendariz rings. Note that the IFP ring property and the Armendariz ring property don't imply each other in general.

The following example shows that there exist RIP rings which are not onesided idempotent reflexive.

Example 2.2. Let $F$ be a field of characteristic zero and $A=F\langle a, b, c\rangle$ be the free algebra with three non-commuting indeterminates $a, b, c$ over $F$.

(1) Due to [12, Example 3.3], let $I$ be the ideal of $A$ generated by

$$
a A b, a^{2}-a
$$

and $R=A / I$. Then $R$ is a right idempotent reflexive ring but not left idempotent reflexive by the computation in [12, Example 3.3]. Note that $R$ is an RIP ring since $R$ is right idempotent reflexive.

(2) Due to [12, Example 3.3], let $I$ be the ideal of $R$ generated by

$$
a A b, b^{2}-b
$$

and $R=A / I$. Then $R$ is left idempotent reflexive but not right idempotent reflexive by the computation in [12, Example 3.3]. Note that $R$ is an RIP ring since $R$ is left idempotent reflexive.

The classes of RIP rings and NI rings do not contain each other by the following example.

Example 2.3. (1) Let $F$ be a field and $R=\left(\begin{array}{cc}F & F \\ 0 & F\end{array}\right)$. Then $R$ is a 2-primal ring and so an NI ring. For $E_{11}, E_{22} \in I d(R)$, we have $E_{22} R E_{11}=0$ but $E_{11} R E_{22} \neq 0$. Thus, $R$ is not RIP.

We also see that the class of RIP rings is not closed under subrings: Indeed, $R=\left(\begin{array}{cc}F & F \\ 0 & F\end{array}\right)$ is a subring of $\operatorname{Mat}_{2}(F)$ which is a reflexive ring (and so an RIP ring) by $[12$, Theorem $2.6(2)]$.

(2) Let $K$ be a field and $n \geq 2$. Let $R=K\left\langle a, b \mid a^{n}=0\right\rangle$ be the free algebra with two non-commuting indeterminates $a, b$ over $K$ with $a^{n}=0$. Then $R$ is an Armendariz ring such that the set of all nilpotent elements of $R$ is not an ideal by [1, Example 4.8]. Hence, $R$ is RIP but not NI.

For a nonempty subset $X$ of a ring $R$, we write $r_{R}(X)=\{c \in R \mid X c=0\}$ which is called the right annihilator of $X$ in $R$. The left annihilator is defined similarly and denoted by $\ell_{R}(X)$.

Proposition 2.4. For a ring $R$, the following are equivalent:

(1) $R$ is an $R I P$ ring;

(2) For $e \in I d(R), r_{R}(e R) \cap I d(R)=\ell_{R}(R e) \cap I d(R)$;

(3) For any nonempty subsets $E$ and $F$ of $I d(R), E R F=0$ implies $F R E=$ 0 ; 
(4) $I J=0$ implies $J I=0$ for all right ideals $I, J$ of $R$ where $I$ and $J$ are right ideals generated by subsets of $I d(R)$; and

(5) $I J=0$ implies $J I=0$ for all ideals $I, J$ of $R$ where $I$ and $J$ are ideals generated by subsets of $\operatorname{Id}(R)$.

Proof. $(1) \Leftrightarrow(2)$ is shown from the definition.

$(1) \Rightarrow(3)$ Let $E R F=0$ for $E, F \subseteq I d(R)$. Then for any $e \in E, f \in F$, we have $e R f=0$. Since $R$ is RIP, $f R e=0$ and so $F R E=0$.

$(3) \Rightarrow(4)$ Let $I=E R$ and $J=F R$ where $E, F \subseteq I d(R)$. Suppose $I J=0$. Then $E R F \subseteq I J=0$ and so by the condition (3), FRE $=0$ and so $J I=0$.

$(4) \Rightarrow(5)$ Let $I=R E R$ and $J=R F R$ where $E, F \subseteq I d(R)$. Suppose $I J=0$. Then $E R F R \subseteq I J=0$. By the condition (4), FRER $=0$ and so $R F R E R=0$. Hence, $J I=0$.

$(5) \Rightarrow(1)$ Suppose that $e R f=0$ for $e, f \in I d(R)$. Then $\operatorname{Re} R R f R=0$ and so by the condition (5), we have $R f R R e R=0$, entailing that $R$ is an RIP ring.

Proposition 2.5. (1) If $R$ is an RIP ring, then so is eRe for each $e \in \operatorname{Id}(R)$.

(2) Let $R / I$ be an RIP ring for some ideal $I$ of a ring $R$. If $I$ is a reduced ring (possibly without identity), then $R$ is $R I P$.

(3) For a central idempotent e of a ring $R, e R$ and $(1-e) R$ are $R I P$ if and only if $R$ is $R I P$.

Proof. (1) Suppose that $R$ is an RIP ring. Let $f, f^{\prime} \in I d(e R e)$ such that $f(e R e) f^{\prime}=0$. Then $f e=f=e f$ and $f^{\prime} e=f^{\prime}=e f^{\prime}$. Since $R$ is RIP, $f R f^{\prime}=0$ implies $0=f^{\prime} R f=f^{\prime}(e R e) f$. Thus $e R e$ is RIP.

(2) Let $e R f=0$ with $e, f \in I d(R)$. Since $R / I$ is RIP, we have $f R e \subseteq I$, and so $(f R e R)^{2}=0$ implies $f R e=0$ since $I$ is reduced. Hence, $R$ is RIP.

(3) Assume that $e R$ and $(1-e) R$ are RIP. Let $f R f^{\prime}=0$ for $f, f^{\prime} \in I d(R)$. Then $e f R f^{\prime}=0$ and $(1-e) f R f^{\prime}=0$, and so $e f^{\prime} R f=0$ and $(1-e) f^{\prime} R f=0$ by assumption. Thus, $f^{\prime} R f=e f^{\prime} R f+(1-e) f^{\prime} R f=0$, proving that $R$ is RIP. The converse is trivial by the result (1).

If $\operatorname{Mat}_{n}(R)$ is an RIP ring, then $R$ is an RIP ring by Proposition 2.5(1), since $R \cong R E_{11}=E_{11} \operatorname{Mat}_{n}(R) E_{11}$. But we don't know whether the converse is true.

Question. If $R$ is an RIP ring, then is $\operatorname{Mat}_{n}(R)$ RIP?

The condition " $I$ is a reduced ring" in Proposition 2.5(2) cannot be dropped by the following.

Example 2.6. Consider the $\operatorname{ring} R=\left(\begin{array}{cc}F & F \\ 0 & F\end{array}\right)$ where $F$ is a field, in Example 2.3, which is not RIP. The only nonzero proper ideals of $R$ are $I_{1}=\left(\begin{array}{cc}F & F \\ 0 & 0\end{array}\right), I_{2}=$ $\left(\begin{array}{ll}0 & F \\ 0 & F\end{array}\right)$ and $I_{3}=\left(\begin{array}{ll}0 & F \\ 0 & 0\end{array}\right)$, and they are not obviously reduced. But $R / I_{1}$ and $R / I_{2}$ are isomorphic to $F$ and $R / I_{3}=\left\{\left(\begin{array}{ll}a & 0 \\ 0 & c\end{array}\right)+I_{3} \mid a, c \in F\right\}$ is a reduced ring. Therefore each $R / I_{i}$ is RIP for $i=1,2,3$. 
Recall that a ring $R$ is called local if $R / J(R)$ is a division ring. A ring $R$ is called semilocal if $R / J(R)$ is semisimple Artinian, and $R$ is called semiperfect if $R$ is semilocal and idempotents can be lifted modulo $J(R)$. Local rings are Abelian and semilocal.

Proposition 2.7. (1) Let $R_{\lambda}(\lambda \in \Lambda)$ be rings. The following are equivalent:

(i) $R_{\lambda}$ is $R I P$ for each $\lambda \in \Lambda$

(ii) The direct product of $R_{\lambda}(\lambda \in \Lambda)$ is RIP.

(iii) The direct sum (possibly without identity) of $R_{\lambda}(\lambda \in \Lambda)$ is $R I P$.

(2) $A$ ring $R$ is Abelian and semiperfect if and only if $R$ is a finite direct sum of local RIP rings.

Proof. (1) (i) $\Rightarrow$ (ii) Assume (i). Let $R$ be the direct product of RIP rings $R_{\lambda}$ $(\lambda \in \Lambda)$. Suppose that $\left(e_{\lambda}\right) R\left(f_{\lambda}\right)=0$ for $\left(e_{\lambda}\right),\left(f_{\lambda}\right) \in I d(R)$. Then $e_{\lambda} R_{\lambda} f_{\lambda}=0$ for each $\lambda \in \Lambda$. Note that $e_{\lambda}, f_{\lambda} \in I d\left(R_{\lambda}\right)$ for each $\lambda \in \Lambda$. Since $R_{\lambda}$ is RIP, $f_{\lambda} R_{\lambda} e_{\lambda}=0$ for each $\lambda \in \Lambda$. Thus $\left(f_{\lambda}\right) R\left(e_{\lambda}\right)=0$ and so $R$ is RIP.

(ii) $\Rightarrow$ (i) Assume (ii). Let $e=\left(e_{\lambda}\right) \in I d(R)$ such that $e_{\lambda}=1$ and $e_{\gamma}=0$ for all $\gamma \neq \lambda$. Then $e R e \cong R_{\lambda}$, and hence $R_{\lambda}$ is RIP by Proposition $2.5(1)$.

(i) $\Leftrightarrow$ (iii) It is similar to the above.

(2) Suppose that $R$ is Abelian and semiperfect. Since $R$ is semiperfect, $R$ has a finite orthogonal set $\left\{e_{1}, e_{2}, \ldots, e_{n}\right\}$ of local idempotents whose sum is 1 by [14, Proposition 3.7.2], say $R=\sum_{i=1}^{n} e_{i} R$ such that each $e_{i} R e_{i}$ is a local ring. Since $R$ is Abelian, each $e_{i} R$ is ideals of $R$ with $e_{i} R=e_{i} R e_{i}$. But each $e_{i} R$ is also an RIP ring by Proposition 2.5(1) since $R$ is Abelian.

Conversely assume that $R$ is a finite direct sum of local RIP rings. Then $R$ is Abelian and semiperfect since local rings are both Abelian and semiperfect.

The class of RIP rings is not closed under homomorphic images.

Example 2.8. Let $K$ be a field and $R=K\langle a, b\rangle$. Then $R$ is reduced and so reflexive. Let $I$ be the ideal of $R$ generated by

$$
a R b, a^{2}-a \text { and } b^{2}-b .
$$

Then $a R b \subseteq I$, but $b a \in b R a \nsubseteq I$ for $a, b \in I d(R / I)$. We conclude that $R / I$ is not RIP.

Example 2.9. Let $R$ be any ring and $n \geq 2$. Then the $n$ by $n$ upper triangular matrix ring $U_{n}(R)$ is not an RIP ring. For $E_{11}, E_{n n} \in \operatorname{Id}\left(U_{n}(R)\right)$, we have $E_{n n} U_{n}(R) E_{11}=0$ but $E_{11} U_{n}(R) E_{n n}=E_{11}\left(R E_{1 n}+R E_{2 n}+\cdots+R E_{n n}\right)=$ $R E_{1 n} \neq 0$.

Next let $R$ be any nonzero ring possibly without identity and suppose that there exist nonzero idempotents $e, f \in R$ with relations $e f=e$. Then the $n$ by $n$ upper triangular matrix ring $U_{n}(R)(n \geq 2)$ is not an RIP ring. Let $A_{i j}=e E_{i j}$ and $B_{i j}=f E_{i j}$ for $i, j=1, \ldots, n$. Then $A_{i i}, B_{i i} \in \operatorname{Id}\left(U_{n}(R)\right)$, and we have $B_{n n} U_{n}(R) A_{11}=0$ but $A_{11} U_{n}(R) B_{n n}$ contains $A_{11}\left(B_{1 n}+B_{2 n}+\cdots+B_{n n}\right)=$ $A_{11} B_{1 n}=A_{1 n}=$ ef $E_{\text {in }}=e E_{\text {in }} \neq 0$. 
For a ring $R, e \in I d(R)$ is called right (resp., left) semicentral if er = ere (resp., re $=$ ere) for all $r \in R$. We use $S_{r}(R)$ (resp., $S_{\ell}(R)$ ) and $B(R)$ for the sets of right (resp., left) semicentral idempotents and central idempotents of $R$. Observe that $S_{r}(R) \cap S_{\ell}(R)=B(R)$ and if $R$ is a semiprime ring, then $S_{\ell}(R)=B(R)=S_{r}(R)$.

For a prime ideal $P$ of a ring $R, O(P)=\{a \in R \mid a R b=0$ for some $b \in R \backslash P\}$, and a ring $R$ is called torsion free if $O(P)=0$ for some prime ideal $P$ of $R$.

Proposition 2.10. (1) Every one-sided semicentral idempotent element of an $R I P$ ring $R$ is central.

(2) Let $R$ be an $R I P$ ring. If $R$ is torsion free, then $B(R)=\{0,1\}$.

Proof. (1) $e \in S_{\ell}(R)$ if and only if $(1-e) R e=0$ if and only if $e R(1-e)=0$ if and only if $e \in S_{r}(R)$.

(2) Assume that $O(P)=0$ for some prime ideal $P$ of $R$. Let $e \in B(R)=$ $S_{\ell}(R)$ by $(1)$. Then $(1-e) R e=0$ and thus $e R(1-e)=0$ since $R$ is RIP. If $e \notin P$, then $1-e \in O(P)=\{0\}$, and so $e=1$. If $e \in P$, then $1-e \notin P$, and hence $e \in O(P)=\{0\}$, and so $e=0$.

Recall that a ring $R$ is called right (resp., left) principally quasi-Baer (or simply, right (resp., left) p.q.-Baer) if the right (resp., left) annihilator of a principal right (resp., left) ideal of $R$ is generated by an idempotent, and that $R$ is called p.q.-Baer if it is both left and right p.q.-Baer.

Proposition 2.11. Let $R$ be a right p.q.-Baer ring. Then the following are equivalent:

(1) $R$ is a semiprime ring;

(2) $R$ is a reflexive ring;

(3) $R$ is a right idempotent reflexive ring;

(4) $R$ is a left idempotent reflexive ring;

(5) $R$ is an $R I P$ ring; and

(6) $S_{\ell}(R)=B(R)=S_{r}(R)$.

Proof. $(1) \Leftrightarrow(2) \Leftrightarrow(3) \Leftrightarrow(4) \Leftrightarrow(6)$ by [12, Proposition 3.15], (4) $\Rightarrow(5)$ by definition and $(5) \Rightarrow(6)$ by Proposition $2.10(1)$.

Corollary 2.12. Let $R$ be an RIP ring. Then $R$ is a right p.q.-Baer ring if and only if $R$ is a p.q.-Baer ring.

Proof. Every right p.q.-Baer and RIP ring $R$ is one-sided idempotent reflexive by Proposition 2.11. Thus $R$ is left p.q.-Baer by [12, Proposition 3.16].

A ring $R$ is usually called directly finite if $a b=1$ implies $b a=1$ for $a, b \in R$. Note that both NI rings and Abelian rings are directly finite by [7, Proposition $2.7(1)]$ and [15, Lemma 3.4], respectively. As in Example 2.3, $\left(\begin{array}{cc}F & F \\ 0 & F\end{array}\right)$, where $F$ is a field, is not RIP but directly finite. In the following we see RIP rings which are not directly finite. 
Example 2.13. (1) Let $R$ be the ring of column finite infinite matrices over a field. Then $R$ is von Neumann regular and so semiprime (hence RIP), but $R$ is not directly finite as can be seen by the matrices $A=E_{12}+E_{23}+\cdots+$ $E_{n(n+1)}+\cdots$ and $B=E_{21}+E_{32}+\cdots+E_{(n+1) n}+\cdots$ such that $A B=1$ but $B A=E_{22}+E_{33}+\cdots+E_{(n+1)(n+1)}+\cdots \neq 1$.

(2) Shepherdson constructed in [21] a domain $D$ such that $M_{2}(D)$ is not directly finite. But $M_{2}(D)$ is prime (hence RIP).

Note. Let $K=\mathbb{Z}_{2}$ and $A=K\langle a, b\rangle$ be the free algebra generated by the noncommuting indeterminates $a, b$ over $K$. Let $I$ be the ideal of $A$ generated by $a b-1$. Set $R=A / I$ and identify $a$ and $b$ with their images in $R$ for simplicity. Then $R$ has the relations $a b=1$. It is obviously true that $R$ is not directly finite.

$K[x, y]$ denotes the polynomial ring with indeterminates over $K$. Every element in $R$ is expressed by

$$
k+a^{i} f_{0}(a)+b^{j} f_{1}(b)+b^{m} f_{2}(a, b),
$$

where $k \in K, i, j, m \geq 1, f_{0}(x), f_{1}(x) \in K[x], f_{2}(x, y) \in K[x, y]$, and every nonzero non-constant monomial of $f_{2}(a, b)$ is of the form $b^{s} a^{t}(s \geq 0$ and $a \geq 1)$. Let $0 \neq e=k+a^{i} f_{0}(a)+b^{j} f_{1}(b)+b^{m} f_{2}(a, b)$ be such that $e^{2}=e$.

Case 1. $k=0$, i.e., $e=a^{i} f_{0}(a)+b^{j} f_{1}(b)+b^{m} f_{2}(a, b)$.

In this case we first have

$$
\begin{aligned}
a^{i} f_{0}(a) & =a^{i} f_{0}(a)\left(a^{i} f_{0}(a)+b^{j} f_{1}(b)+b^{m} f_{2}(a, b)\right) \\
& =f_{0}(a)\left(a^{2 i} f_{0}(a)+a^{i} b^{j} f_{1}(b)+a^{i} b^{m} f_{2}(a, b)\right) .
\end{aligned}
$$

Here assume $f_{0}(a) \neq 0$. Then $f_{1}(b)=0$ and $e=a^{i} f_{0}(a)+b^{j} f_{1}(b)+b^{m} f_{2}(a, b)$. But $e^{2}=e$ yields $f_{2}(a, b)=0$, entailing $e=a^{i} f_{0}(a)$. We also get $e=a^{i} f_{0}(a)=$ 0 from $e^{2}=e$, a contradiction. So $a^{i} f_{0}(a)=0$, i.e., $e=b^{j} f_{1}(b)+b^{m} f_{2}(a, b)$. Similar computation gives $b^{j} f_{1}(b)=0$, so $e=b^{m} f_{2}(a, b)$. Then we have $b^{m} f_{2}(a, b)=e=e^{2}=b^{m} f_{2}(a, b) b^{m} f_{2}(a, b)$. Thus we can conclude that $e=b^{m} f_{2}(a, b)$ is of the form

$$
e=\sum_{\text {finite }} b^{s} a^{t} \text { for } s, t \geq 1
$$

Case 2. $k=1$, i.e., $e=1+a^{i} f_{0}(a)+b^{j} f_{1}(b)+b^{m} f_{2}(a, b)$.

In this case we first have $1+f+f+f^{2}=e^{2}=e=1+f$, where $f=$ $a^{i} f_{0}(a)+b^{j} f_{1}(b)+b^{m} f_{2}(a, b)$. Then $f+f^{2}=0$, so $f=-f^{2}=-(-f)^{2}$ and $(-f)^{2}=-f$. By the computation in the case 1 , we have $-f$ is of the form $\sum_{\text {finite }} b^{s} a^{t}$ for $s, t \geq 1$. Thus $e$ is of the form

$$
e=1-\sum_{\text {finite }} b^{s} a^{t} \text { for } s, t \geq 1,
$$

where it is understood that $a^{0}=b^{0}=1$. 
Therefore the set of all idempotents in $R$ is

$$
\left\{0,1, \sum_{\text {finite }} b^{s} a^{t}, 1-\sum_{\text {finite }} b^{s} a^{t} \text { for } s, t \geq 1\right\} .
$$

For example, we can see $b^{i-1} a^{i-1}-b^{i} a^{i}$ (for $i \geq 1$ ) to be found by Jacobson in [8]. Here we raise the following two questions:

(1) What are more exact expressions of the idempotents $\sum_{\text {finite }} b^{s} a^{t}$ ?

(2) Is the ring $R$ RIP?

\section{Extensions of RIP rings}

In this section we observe the reflexive-idempotents-property of various kinds of ring extensions. We first consider the cases of polynomial rings and power series rings. The following includes the result of [12, Theorem 3.13].

Theorem 3.1. (1) $R$ is an $R I P$ ring if and only if $R[x]$ is an $R I P$ ring.

(2) $R$ is an $R I P$ ring if and only if $R[[x]]$ is an $R I P$ ring.

(3) $R$ is a right (resp., left) idempotent reflexive ring if and only if $R[x]$ is a right (resp., left) idempotent reflexive ring.

(4) $R$ is a right (resp., left) idempotent reflexive ring if and only if $R[[x]]$ is a right (resp., left) idempotent reflexive ring.

Proof. We apply the method in the proof of [12, Theorem 3.13].

(1) Suppose that $R$ is an RIP ring. Note that $f(x) R[x] g(x)=0$ if and only if $f(x) R g(x)=0$ for $f(x), g(x) \in R[x]$. Let $e(x)=\sum_{i=0}^{m} e_{i} x^{i}, f(x)=$ $\sum_{j=0}^{n} f_{j} x^{j} \in I d(R[x])$ such that $e(x) R f(x)=0$. From $e^{2}(x)=e(x)$, we have

$$
e_{0}^{2}=e_{0}, e_{h}=\sum_{u+v=h} e_{u} e_{v} \text { for } h=1,2, \ldots, m
$$

In this situation, note that $e_{h} \in\left(e_{0}, \ldots, e_{h-1}\right)$ for all $h$ where $\left(e_{0}, \ldots, e_{h-1}\right)$ denotes the ideal of $R$ generated by $e_{0}, \ldots, e_{h-1}$. Thus $e_{h} \in\left(e_{0}\right)$ inductively for all $h=0,1, \ldots, m$, where $\left(e_{0}\right)=R e_{0} R$. Similarly we have $f_{k} \in\left(f_{0}\right)$ for all $k=0,1, \ldots, n$.

If $e_{0}=0$, then $e(x)=0$ since every $e_{h}$ is contained in $\left(e_{0}\right)$, entailing $f(x) R e(x)=0$. So assume $e_{0} \neq 0$. From $e(x) R f(x)=0$, we get $e_{0} R f_{0}=$ 0 . Since $R$ is RIP and $e_{0}, f_{0} \in I d(R)$, we have $f_{0} R e_{0}=0$. This yields $\left(R f_{0} R\right) R\left(R e_{0} R\right)=0$. But $e_{i} \in R e_{0} R$ and $f_{j} \in R f_{0} R$ for all $i, j$. This implies $f_{j} R e_{i}=0$ for all $i, j$, entailing $f(x) R[x] e(x)=0$. We conclude that $R[x]$ is RIP.

Conversely, suppose that $R[x]$ is RIP. Let $e R f=0$ for $e, f \in I d(R)$. Then $e R[x] f=0$, and so $f R[x] e=0$ and $f R e=0$. Thus $R$ is RIP.

(2) The proof is similar by much to the proof of (1).

(3) The proof for an left idempotent reflexive ring is similar to the proof of (1), and the case of a right idempotent reflexive ring is also obtained symmetrically but we write it for completeness. Suppose that $R$ is a right idempotent reflexive ring. Let $f(x)=\sum_{i=0}^{m} a_{i} x^{i}, e^{2}(x)=e(x)=\sum_{j=0}^{n} e_{j} x^{j}, \in R[x]$ such 
that $f(x) R e(x)=0$. By the proof of $(1)$, we have $e_{h} \in\left(e_{0}\right)$ inductively for all $h=0,1, \ldots, n$. We assume $e_{0} \neq 0$. From $f(x) R e(x)=0$, we get $a_{0} R e_{0}=0$ and $a_{0} R e_{0} R=0$. This yields $a_{0} R e_{h}=0$ (equivalently, $a_{0} R e(x)=0$ ) for all $h=0,1, \ldots, n$ since $e_{h} \in\left(e_{0}\right)$ for all $h$. Consequently $\left(\sum_{i=1}^{m} a_{i} x^{i}\right) \operatorname{Re}(x)=0$. Then $a_{1} R e_{0}=0$, and we also have $a_{1} R e(x)=0$ similarly. Proceeding in this manner, we inductively obtain $a_{i} R e(x)=0$ for all $i=0,1, \ldots, m$. This implies that

$$
a_{i} R e_{j}=0 \text { for all } i=0,1, \ldots, m \text { and } j=0,1, \ldots, n \text {. }
$$

Especially, $a_{i} R e_{0}=0$ for all $i=0,1, \ldots, m$. Since $R$ is right idempotent reflexive and $e_{0} \in I d(R)$, we have $e_{0} R a_{i}=0$ (equivalently, $e_{0} R f(x)=0$ ) for all $i=0,1, \ldots, m$. This yields $\operatorname{Re}_{0} R f(x)=0$. But $e_{j} \in R e_{0} R$ for all $j=0,1, \ldots, n$. This yields $e_{j} R a_{i}=0$ for all $i, j$, entailing $e(x) R[x] f(x)=0$. We conclude that $R[x]$ is right idempotent reflexive.

The converse can be shown by the similar argument to the converse proof of (1).

(4) The proof is similar by much to the proof of (3).

Let $R$ be an algebra over a commutative ring $S$. Following Dorroh [5], the Dorroh extension of $R$ by $S$ is the Abelian group $R \oplus S$ with multiplication given by $\left(r_{1}, s_{1}\right)\left(r_{2}, s_{2}\right)=\left(r_{1} r_{2}+s_{1} r_{2}+s_{2} r_{1}, s_{1} s_{2}\right)$ for $r_{i} \in R$ and $s_{i} \in S$.

Theorem 3.2. Let $R$ be an algebra over a commutative ring $S$. Then $R$ is $R I P$ if and only if the Dorroh extension $D$ of $R$ by $S$ is $R I P$.

Proof. In the following computations, $s \in S$ is identified with $s 1 \in R$. Note that $R=\{r+s \mid(r, s) \in D\}$, where $s=s 1$.

Suppose that $R$ is RIP. Let $\left(e_{1}, s_{1}\right) D\left(e_{2}, s_{2}\right)=0$ for $\left(e_{1}, s_{1}\right),\left(e_{2}, s_{2}\right) \in \operatorname{Id}(D)$. Since $\left(e_{i}, s_{i}\right)=\left(e_{i}^{2}+2 s_{i} e_{i}, s_{i}^{2}\right)$, we have that $\left(e_{i}+s_{i} 1\right)^{2}=e_{i}^{2}+2 s_{i} e_{i}+s_{i}^{2} 1=e_{i}+$ $s_{i} 1$ is an idempotent in $R$. Since $\left(e_{1}, s_{1}\right)(r, 0)\left(e_{2}, s_{2}\right)=\left(e_{1} r e_{2}+s_{1} r e_{2}+s_{2} e_{1} r+\right.$ $\left.s_{1} s_{2} r, 0\right)=(0,0)$ and $\left(e_{1}+s_{1} 1\right) r\left(e_{2}+s_{2} 1\right)=e_{1} r e_{2}+s_{1} r e_{2}+s_{2} e_{1} r+s_{1} s_{2} r$, we have $\left(e_{1}+s_{1} 1\right) R\left(e_{2}+s_{2} 1\right)=0$. Since $R$ is RIP, we get $\left(e_{2}+s_{2} 1\right) R\left(e_{1}+s_{1} 1\right)=0$. Hence $e_{2} r e_{1}+s_{2} r e_{1}+s_{1} e_{2} r+s_{1} s_{2} r=0$ for all $r \in R$. Let $(r, s) \in D$. Then

$$
\begin{aligned}
\left(e_{2}, s_{2}\right)(r, s)\left(e_{1}, s_{1}\right) & =\left(e_{2}(r+s 1) e_{1}+s_{2}(r+s 1) e_{1}+s_{1} e_{2}(r+s 1)+s_{1} s_{2} r, s_{2} s s_{1}\right) \\
& =\left(-s_{1} s_{2} s 1, s_{1} s s_{2}\right)=(0,0)
\end{aligned}
$$

where the last equality follows because $\left(e_{1}, s_{1}\right) D\left(e_{2}, s_{2}\right)=0$ implies that $s_{1} s s_{2}$ $=0$ for all $s \in S$. Therefore $D$ is RIP.

Suppose now that $D$ is RIP. Let $e R f=0$ for $e, f \in I d(R)$. Then

$$
(e, 0)(r, s)(f, 0)=(e(r+s 1) f, 0)=(0,0)
$$

for all $(r, s) \in D$. Since $D$ is RIP, we have that $(f, 0) D(e, 0)=0$. In particular, $(f, 0)(r, s)(e, 0)=($ fre, 0$)=(0,0)$ for all $r \in R$. Therefore $f R e=0$ and thus $R$ is RIP. 
For a ring $R$ and $n \geq 2$, let

$$
\begin{gathered}
V_{n}(R)=\left\{m=\left(m_{i j}\right) \in D_{n}(R) \mid m_{s t}=m_{(s+1)(t+1)} \text { for } s=1, \ldots, n-2\right. \text { and } \\
t=2, \ldots, n-1\} .
\end{gathered}
$$

For any $\operatorname{ring} R$ and $n \geq 2$, the $n$ by $n$ upper triangular matrix $\operatorname{ring} U_{n}(R)$ is not RIP by Example 2.9; while $\operatorname{Mat}_{n}(R), D_{n}(R)$ and $V_{n}(R)$ over a reflexive ring $R$ are right idempotent reflexive rings by [12, Theorem 2.6(2) and Theorem 3.9], and so they are RIP rings. Moreover, we have the following.

Theorem 3.3. Let $R$ be a ring and $n \geq 2$.

(1) $R$ is an $R I P$ ring if and only if $\bar{D}_{n}(R)$ is an $R I P$ ring.

(2) $R$ is an $R I P$ ring if and only if $V_{n}(R)$ is an $R I P$ ring.

(3) $R$ is a right (resp., left) idempotent reflexive ring if and only if $D_{n}(R)$ a right (resp., left) idempotent reflexive ring.

(4) $R$ is a right (resp., left) idempotent reflexive ring if and only if $V_{n}(R)$ is a right (resp., left) idempotent reflexive ring.

Proof. We apply the method in the proof of [12, Theorem 3.9].

(1) Note that if $E^{2}=E=\left(e_{i j}\right) \in D_{n}(R)$ with $e_{i i}=e$ for all $i=1, \ldots, n$, then $e_{i j} \in R e R$ by the proof of [12, Theorem 3.9]. Suppose that $E D_{n}(R) F=0$ for $E=\left(e_{i j}\right), F=\left(f_{k l}\right) \in I d\left(D_{n}(R)\right)$. Let $D=\left(d_{u v}\right)$ be any in $D_{n}(R)$. Set $e_{i i}=e, d_{u u}=d, f_{k k}=f$ for all $i, u, k=1, \ldots, n$. Then $e, f \in I d(R)$. Note that $d$ runs over $R$. Then $e d f=0$ for all $d \in R$ and hence $e R f=0$. Since $R$ is RIP and $e, f \in I d(R)$, we have $f R e=0$. This yields $(R f R) R(R e R)=0$. But $e_{i j} \in R e R$ and $f_{k l} \in R f R$ for all $i, j, k, l$. This implies $f_{k l} R e_{i j}=0$ for all $i, j, k, l$, entailing $F D_{n}(R) E=0$ and so $D_{n}(R)$ is RIP.

Conversely, assume that $D_{n}(R)$ is an RIP ring. Let $e R f=0$ for $e, f \in I d(R)$. We have $E D_{n}(R) F=0$ for $E=e \sum_{i=1}^{n} E_{i i}, F=f \sum_{i=1}^{n} E_{i i} \in I d\left(D_{n}(R)\right)$, and thus $F D_{n}(R) E=0$ by assumption. This entails $f R e=0$ and therefore $R$ is RIP.

(2) is the same as the proof of (1).

(3) The proof for a right idempotent reflexive ring is similar to the proof of (1), but we write it for completeness. Suppose that $A D_{n}(R) E=0$ for $A=\left(a_{i j}\right), E^{2}=E=\left(e_{k l}\right) \in D_{n}(R)$. Let $D=\left(d_{u v}\right)$ be any in $D_{n}(R)$. Set $a_{i i}=a, d_{u u}=d, e_{k k}=e=e^{2}$ for all $i, u, k=1, \ldots, n$. First, we get ade $=0$ for all $d \in R$. So, we have $a d e_{k l}=0$ for all $d \in R$, since $a R e R=0$ and $e_{k l} \in R e R$ as noted in the proof of (1). By these two results, we can also obtain $a_{i j} d e=0$ through an induction on $j-i$. Since $R$ is right idempotent reflexive and $e \in I d(R)$, we also get $e R a_{i j}=0$ for all $i, j$, and then $R e R a_{i j}=0$. But $e_{k l} \in R e R$ for any $k, j$. Then $e_{k l} R a_{i j}=0$ for all $i, j, k, l$. This yields $E D_{n}(R) A=0$. Therefore $D_{n}(R)$ is a right idempotent reflexive ring for $n \geq 2$.

The converse can be shown by the similar argument to the converse proof of (1), and the proof for an left idempotent reflexive ring is symmetrically obtained to the above.

(4) is the same as the proof of (1). 
Recall that for a ring $R$ and an $(R, R)$-bimodule $M$, the trivial extension of $R$ by $M$ is the $\operatorname{ring} T(R, M)=R \oplus M$ with the usual addition and the following multiplication: $\left(r_{1}, m_{1}\right)\left(r_{2}, m_{2}\right)=\left(r_{1} r_{2}, r_{1} m_{2}+m_{1} r_{2}\right)$. This is isomorphic to the ring of all matrices $\left(\begin{array}{cc}r & m \\ 0 & r\end{array}\right)$, where $r \in R$ and $m \in M$ and the usual matrix operations are used.

Corollary 3.4. (1) A ring $R$ is $R I P$ (right (left) idempotent reflexive) if and only if the trivial extension $T(R, R)$ is RIP (right (left) idempotent reflexive).

(2) A ring $R$ is RIP (right (left) idempotent reflexive ring) if and only if $R[x] /\left(x^{n}\right)$ is an $R I P$ (right (left) idempotent reflexive) ring for any positive integer $n$, where $\left(x^{n}\right)$ is an ideal of $R[x]$ generated by $x^{n}$.

Proof. It follows directly from Theorem 3.3 and the fact $V_{n}(R) \cong R[x] /\left(x^{n}\right)$ by $[16]$.

Recall that an element $u$ of a ring $R$ is right regular if $u r=0$ implies $r=0$ for $r \in R$. Similarly, left regular elements can be defined. An element is regular if it is both left and right regular (and hence not a zero divisor).

Proposition 3.5. Let $M$ be a multiplicatively closed subset of a ring $R$ consisting of central regular elements.

(1) If $M^{-1} R$ is RIP, then $R$ is RIP.

(2) Suppose that every idempotent in $M^{-1} R$ is of the form $u^{-1} e$ with $e \in$ $I d(R)$ and $u \in M$. If $R$ is $R I P$, then $M^{-1} R$ is $R I P$.

Proof. (1) Suppose that $M^{-1} R$ is RIP. Let $e R f=0$ for $e, f \in I d(R)$. For any $r \in R$ with $w \in M, 0=w^{-1} e r f=e\left(w^{-1} r\right) f$. So we have $e\left(M^{-1} R\right) f=0$ and so $f\left(M^{-1} R\right) e=0$, since $M^{-1} R$ is RIP. Thus $f R e=0$, showing that $R$ is RIP.

(2) Suppose that $R$ is RIP. Let $\alpha\left(M^{-1} R\right) \beta=0$ where $\alpha=u^{-1} e, \beta=v^{-1} f \in$ $I d\left(M^{-1} R\right)$ with $e, f \in I d(R)$ and $u, v \in M$. Since $M$ is contained in the center of $R$, we have $0=\left(u^{-1} e\right)\left(w^{-1} r\right)\left(v^{-1} f\right)=(u w v)^{-1}(e r f)$ for any $w^{-1} r \in M^{-1} R$, and so $e R f=0$. Since $R$ is RIP, $f R e=0$ and hence $(u w v)^{-1}(f r e)=0$ for any $r \in R$. This shows that $\beta\left(M^{-1} R\right) \alpha=0$, concluding that $M^{-1} R$ is RIP.

In connection with the converse of Proposition 3.5(1), it is not true that there exist $e \in I d(R)$ and $u \in M$ such that $\alpha=u^{-1} e$, whenever $\alpha \in I d\left(M^{-1} R\right)$. For example, consider the $\operatorname{ring} R=K[x ; y] / I$, where $K$ is a field and $I$ is the ideal of the polynomial ring $K[x, y]$ generated by $x^{2}-x y$. We denote by $\overline{p(x, y)}$ the image of $p(x, y) \in K[x, y]$ under the natural projection $K[x, y] \rightarrow R$. It is easy to see that $\bar{y}$ is a regular element in $R$. Let $\Delta=\left\{\overline{y^{n}} \mid n \geq 0\right\} \subseteq R$. Then in $M^{-1} R$ the element $\bar{y}^{-1} \bar{x}$ is an idempotent, but $x$ is not an idempotent in $R$. Note that $\overline{y^{n}} \bar{x}$ is not an idempotent in $R$ for $n \geq 0$.

The ring of Laurent polynomials in $x$, coefficients in a ring $R$, consists of all formal sums $\sum_{i=k}^{n} r_{i} x^{i}$ with obvious addition and multiplication, where $r_{i} \in R$ and $k, n$ are (possibly negative) integers with $k \leq n$. We denote this ring by $R\left[x ; x^{-1}\right]$. 
Corollary 3.6. Let $R$ be a ring and suppose that every idempotent in $R\left[x ; x^{-1}\right]$ is of the form $f(x) x^{m}$ for some $f(x) \in \operatorname{Id}(R[x])$ and $m \in \mathbb{Z}$. Then the following conditions are equivalent:

(1) $R$ is $R I P$;

(2) $R[x]$ is RIP; and

(3) $R\left[x ; x^{-1}\right]$ is $R I P$.

Proof. $(1) \Leftrightarrow(2)$ comes from Theorem 3.1(1), and $(2) \Leftrightarrow(3)$ follows from Proposition 3.5 letting $M=\left\{1, x, x^{2}, \ldots\right\}$.

Consider the idempotent $f(x) x^{m}$ in the assumption of Corollary 3.6 for the case of $m \geq 1$. Let $f(x)=a_{0}+\cdots+a_{l} x^{l}$. Then $f(x)^{2} x^{2 m}=f(x) x^{m}$ yields $f(x)=f(x)^{2} x^{m}$, and thus $a_{0}=0$, i.e., $f(x)=a_{1} x+\cdots+a_{l} x^{l}$. Next we have $a_{1}=0$ by the same computation, and so we inductively obtain $f(x)=0$. Thus one may investigate the case of $m \leq-1$ to find the structure of the idempotent $f(x) x^{m}$ in $R\left[x ; x^{-1}\right]$.

A ring $R$ is called right (resp., left) Ore if given $a, b \in R$ with $b$ regular there exist $a_{1}, b_{1} \in R$ with $b_{1}$ regular such that $a b_{1}=b a_{1}$ (resp., $b_{1} a=a_{1} b$ ). It is a well-known fact that $R$ is a right (resp., left) Ore ring if and only if the classical right (resp., left) quotient ring of $R$ exists. Suppose that there exists the classical right quotient ring $Q(R)$ of a ring $R$. If $R$ is reflexive, then so is the right quotient ring $Q(R)$ by [12, Theorem 2.11], but we do not know whether $Q(R)$ is RIP when $R$ is RIP. Recall that every IFP ring is RIP. However, we have the following related fact.

Remark 3.7. Suppose that a ring $R$ is right Ore with the classical right quotient ring $Q(R)$, and that $R$ is IFP. Then we obtain that $a u^{-1} Q(R) b v^{-1}=0$ for $a u^{-1}, b v^{-1} \in Q(R)$ implies $a R b=0$. Let $a u^{-1} Q(R) b v^{-1}=0$ for $a u^{-1}, b v^{-1} \in$ $Q(R)$. Then there exist $c \in R$ and regular $w \in R$ such that $u^{-1} b=c w^{-1}$ by [19, Proposition 2.1.16]. This yields $a c w^{-1}=0$ and $a c=0$. But since $R$ is IFP, $a R c=0$ and $a R u^{-1} b=a R c w^{-1}=0$. Especially we obtain $a b=a u u^{-1} b=0$. This implies $a R b=0$.

\section{RIP rings of minimal order}

$\mathrm{Xu}$ and Xue [22, Theorem 8] proved that a noncommutative IFP ring with identity of minimal order is a local ring of order 16 and if $R$ is such a ring, then $R \cong R_{i}$ for some $i \in\{1,2,3,4,5\}$, where $R_{i}$ 's are the rings in the following example.

Example 4.1. We have five kinds of noncommutative finite Abelian rings with 16 elements by the help of [22, Example 7].

(1) Let $R_{1}=G F(2)[x, y] / I$, where $G F(2)[x, y]$ is the polynomial ring over $G F(2)$ with non-commuting indeterminates $x, y$ and $I$ is the ideal of $G F(2)[x, y]$ generated by $x^{3}, y^{3}, y x, x^{2}-x y, y^{2}-x y$. 
(2) Let $R_{2}=\mathbb{Z}_{4}\langle x, y\rangle / I$, where $\mathbb{Z}_{4}\langle x, y\rangle$ is the free algebra with non-commuting indeterminates $x, y$ over $\mathbb{Z}_{4}$ and $I$ is the ideal of $\mathbb{Z}_{4}\langle x, y\rangle$ generated by $x^{3}, y^{3}, y x, x^{2}-x y, x^{2}-2, y^{2}-2,2 x, 2 y$.

(3) Let $R_{3}=\left\{\left(\begin{array}{cc}a & b \\ 0 & a^{2}\end{array}\right) \mid a, b \in G F\left(2^{2}\right)\right\}$.

(4) Let $R_{4}=G F(2)[x, y] / I$, where $I$ is the ideal of $G F(2)[x, y]$ generated by $x^{3}, y^{2}, y x, x^{2}-x y$. $R_{4}$ is isomorphic to $D_{3}\left(\mathbb{Z}_{2}\right)$ through the corresponding $x \mapsto E_{12}+E_{23}$ and $y \mapsto E_{23}$.

(5) Let $R_{5}=\mathbb{Z}_{4}\langle x, y\rangle / I$, where $I$ is the ideal of $\mathbb{Z}_{4}\langle x, y\rangle$ generated by $x^{3}, y^{2}, y x, x^{2}-x y, x^{2}-2,2 x, 2 y$.

Eldridge proved that if a finite ring has a cube free factorization, then it is commutative [6, Theorem], and that if a ring $A$ is of order $p^{3}, p$ a prime, then $A \cong U_{2}(G F(p))[6$, Proposition]. Thus every noncommutative ring of minimal order is isomorphic to $U_{2}\left(\mathbb{Z}_{2}\right)$. However, $U_{2}\left(\mathbb{Z}_{2}\right)$ is not RIP by Example 2.9, and so an RIP ring of minimal order has order $\geq 16$. But an RIP ring of minimal order must have order 16 , considering the semiprime $\operatorname{ring} \operatorname{Mat}_{2}\left(\mathbb{Z}_{2}\right)$ and the rings of Example 4.1.

Observe that every $R_{i}$ in Example 4.1 is Abelian. Hence, if $R$ is a noncommutative Abelian RIP ring of minimal order, then $R$ is of order 16 such that $R$ is isomorphic to $R_{i}$ for some $i \in\{1,2,3,4,5\}$ in Example 4.1.

Moreover, $\operatorname{Mat}_{2}\left(\mathbb{Z}_{2}\right)$ is reflexive by $[12$, Theorem 2.6] and hence RIP. Therefore,

Theorem 4.2. If $R$ is a non-Abelian RIP ring of minimal order, then $R$ is of order 16 and is isomorphic to $\operatorname{Mat}_{2}\left(\mathbb{Z}_{2}\right)$.

Proof. Let $R$ be a non-Abelian RIP ring of minimal order. Then it is true that $R$ cannot be local since local rings are Abelian. By the Wedderburn-Artin theorem, $R / J(R) \cong \sum_{i=1}^{n} \operatorname{Mat}_{k_{i}}\left(D_{i}\right)$ for some $k_{i}$ 's and fields $D_{i}$ 's. Here assume that $k_{i}=1$ for all $i$. Then we have there cases of $|J(R)|=2,|J(R)|=4$, and $|J(R)|=8$.

If $|J(R)|=8$, then $R / J(R) \cong \mathbb{Z}_{2}$ and so $R$ is local, a contradiction. Thus $|J(R)|=2$ or $|J(R)|=4$.

Let $|J(R)|=4$. Then $R / J(R) \cong \mathbb{Z}_{2} \oplus \mathbb{Z}_{2}$. Since $J(R)$ is nilpotent, there exist orthogonal nonzero idempotents $e_{1}, e_{2}$ with $e_{1}+e_{2}=1$ (i.e., $e_{2}=1-e_{1}$ ) by [14, Proposition 3.7.2], and moreover we have

$$
R=\{x+y \mid x \in \operatorname{Id}(R), y \in J(R)\},
$$

where $\operatorname{Id}(R)=\left\{0,1, e_{1}, e_{2}\right\}$. Suppose that $e R f=0$ for $e, f \in I d(R)$. Then $e$ and $f$ are orthogonal each other, say $e=e_{1}, f=e_{2}$. Let $r=x+y \in R$ with $x \in I d(R)$ and $y \in J(R)$. Then $0=e_{1} r e_{2}=e_{1}(x+y) e_{2}=e_{1} y e_{2}$ since $e_{1} x e_{2}=0$. But since $R$ is RIP, $e_{2} R e_{1}=0$ and so we get $e_{2} y e_{1}=0$ since $e_{2} x e_{1}=0$. This entails $r=\left(e_{1}+e_{2}\right) r\left(e_{1}+e_{2}\right)=e_{1} r e_{1}+e_{2} r e_{2}$. Since $R$ is non-Abelian, there exist $g \in\left\{e_{1}, e_{2}\right\}$ and $s \in R$ such that $g s-s g \neq 0$. Note $g s-s g \in J(R)$. Since $g=e_{1}$ or $g=e_{2}$, we have

$$
g s-s g=e_{1}(g s-s g) e_{1}+e_{2}(g s-s g) e_{2}=0,
$$


a contradiction. The case of $e=e_{2}$ and $f=e_{1}$ also induces a contradiction through a similar computation. This implies $|J(R)| \neq 4$.

Let $|J(R)|=2$. Then $R / J(R) \cong \mathbb{Z}_{2} \oplus \mathbb{Z}_{2} \oplus \mathbb{Z}_{2}$. Since $J(R)$ is nilpotent, there exist orthogonal nonzero idempotents $e_{1}, e_{2}, e_{3}$ with $e_{1}+e_{2}+e_{3}=1$ by [14, Proposition 3.7.2], and moreover we have

$$
R=\{x+y \mid x \in I d(R), y \in J(R)\},
$$

where $\operatorname{Id}(R)=\left\{0,1, e_{1}, e_{2}, e_{3}, 1-e_{1}, 1-e_{2}, 1-e_{3}\right\}$. For every $r=x+y \in R$ with $x \in I d(R)$ and $y \in J(R)$, we have $e_{i} r e_{j}=e_{i} y e_{j}$ for $i \neq j$ since $e_{i} x e_{j}=0$. If $e_{i} y e_{j} \neq 0$, then $J(R)=\left\{0, e_{i} y e_{j}\right\}$. Thus $e_{j} J(R) e_{i}=0$, and so $e_{j} R e_{i}=0$ since $e_{j} I d(R) e_{i}=0$. But since $R$ is RIP, we get $e_{i} R e_{j}=0$ and this yields $e_{i} y e_{j}=0$, a contradiction. Therefore we can conclude that

$$
e_{i} R e_{j}=0 \text { for all } i, j \text { with } i \neq j .
$$

Now suppose that $e R f=0$ for $e, f \in I d(R)$. Then $e$ and $f$ are orthogonal each other, say $e=e_{1}$ and $f=e_{2}$. But since $R$ is RIP, $e_{2} R e_{1}=0$ and so we get $e_{2} y e_{1}=0$ since $e_{2} x e_{1}=0$. This entails

$$
r=\left(e_{1}+e_{2}+e_{3}\right) r\left(e_{1}+e_{2}+e_{3}\right)=e_{1} r e_{1}+e_{2} r e_{2}+e_{3} r e_{3} .
$$

Since $R$ is non-Abelian, $e_{k}$ is non-central for some $k \in\{1,2,3\}$. If $e_{1}$ is noncentral, then there exists $s \in R$ such that $e_{1} s-s e_{1} \neq 0$. Note $e_{1} s-s e_{1} \in J(R)$. Then we have

$$
\begin{aligned}
e_{1} s-s e_{1} & =\left(e_{1}+e_{2}+e_{3}\right)\left(e_{1} s-s e_{1}\right)\left(e_{1}+e_{2}+e_{3}\right) \\
& =e_{1}\left(e_{1} s-s e_{1}\right) e_{1}+e_{2}\left(e_{1} s-s e_{1}\right) e_{2}+e_{3}\left(e_{1} s-s e_{1}\right) e_{3}=0,
\end{aligned}
$$

a contradiction. Each case of $\left(e_{2}\right.$ is non-central) and $\left(e_{3}\right.$ is non-central $)$ also induces a contradiction through a similar computation. The computations for other cases of $e$ and $f$ are also similar, inducing contradictions.

Thus we must have $k_{i} \geq 2$ for some $i$. But $R$ is of order 16 and hence we have $R / J(R) \cong \operatorname{Mat}_{2}\left(\mathbb{Z}_{2}\right)$ and $J(R)=0$. This implies $R \cong \operatorname{Mat}_{2}\left(\mathbb{Z}_{2}\right)$.

Observe that $\operatorname{Mat}_{n}\left(\mathbb{Z}_{2}\right)$ is semiprime and so we get the following by Theorem 4.2 .

Corollary 4.3. Let $R$ be a ring. Then $R$ is a non-Abelian RIP ring of minimal order if and only if $R$ is a non-Abelian semiprime ring of minimal order if and only if $R$ is a non-Abelian reflexive ring of minimal order if and only if $R$ is a non-Abelian right idempotent reflexive ring of minimal order if and only if $R$ is a non-Abelian left idempotent reflexive ring of minimal order.

Recall that an IFP ring with identity is Abelian, but the following example shows that this is no longer valid for the case of rings without identity.

Example 4.4. The rings $R_{1}=\left(\begin{array}{cc}G F(p) & G F(p) \\ 0 & 0\end{array}\right)$ and $R_{2}=\left(\begin{array}{cc}G F(p) & 0 \\ G F(p) & 0\end{array}\right)$ are minimal noncommutative IFP rings without identity by $[22$, p. 71$]$ which have 4 
elements, respectively. Notice that they are not Abelian by a simple computation. We will show that $R_{i}$ 's are RIP rings.

The set of all nonzero idempotents in $R_{1}$ is $E=\left\{\left(\begin{array}{ll}1 & d \\ 0 & 0\end{array}\right) \mid d \in G F(p)\right\}$. Suppose $e R_{1} a=0$ for $e \in E$ and $a \in R$. Then $a=0$ since $e R_{1}=R_{1}$, obtaining $a R_{1} e=0$. So $R_{1}$ is left idempotent reflexive, and hence $R_{1}$ is RIP.

The set of all nonzero idempotents in $R_{2}$ is $E=\left\{\left(\begin{array}{ll}1 & 0 \\ d & 0\end{array}\right) \mid d \in G F(p)\right\}$. By a similar computation to above, we can show that $R_{2}$ is right idempotent reflexive and so $R_{2}$ is RIP.

Lemma 4.5 ([13, Lemma 2.7]). Let $R$ be a ring and $N$ be a nil ideal of $R$. If $|N|=4$, then $N$ is a commutative ring with $N^{3}=0$.

Theorem 4.6. Let $R$ be a ring without identity. If $R$ is a non-Abelian RIP ring of minimal order, then $R$ is isomorphic to $\left(\begin{array}{cc}\mathbb{Z}_{2} & \mathbb{Z}_{2} \\ 0 & 0\end{array}\right)$ or $\left(\begin{array}{ll}\mathbb{Z}_{2} & 0 \\ \mathbb{Z}_{2} & 0\end{array}\right)$.

Proof. Let $R$ be a non-Abelian RIP ring of minimal order. Then $|R|=4$ by the existence of the non-Abelian RIP $\operatorname{ring}\left(\begin{array}{cc}\mathbb{Z}_{2} & \mathbb{Z}_{2} \\ 0 & 0\end{array}\right)$ as in Example 4.4. If $R$ is nilpotent then $R$ is commutative by Lemma 4.5, a contradiction. If $|J(R)|=0$, then $R$ is also commutative by the proof of [11, Theorem 1.15], a contradiction. Thus we have the result of $|J(R)|=2$, whence we also follow the proof $[11$, Theorem 1.15] to conclude that $R$ is isomorphic to $\left(\begin{array}{cc}\mathbb{Z}_{2} & \mathbb{Z}_{2} \\ 0 & 0\end{array}\right)$ or $\left(\begin{array}{ll}\mathbb{Z}_{2} & 0 \\ \mathbb{Z}_{2} & 0\end{array}\right)$.

Hence, we have the following by Theorem 4.6.

Corollary 4.7. Let $R$ be a ring without identity. Then $R$ is a noncommutative $R I P$ ring of minimal order if and only if $R$ is a noncommutative IFP ring of minimal order.

Acknowledgments. The authors thank the referee for very careful reading of the manuscript and many valuable suggestions that improved the paper by much. The first named author was supported by Basic Science Research Program through the National Research Foundation of Korea(NRF) funded by the Ministry of Education(No. 2010-0022160) and the second named author was supported by Basic Science Research Program through the National Research Foundation of Korea(NRF) funded by the Ministry of Educa$\operatorname{tion}(201306530001)$.

\section{References}

[1] R. Antoine, Nilpotent elements and Armendariz rings, J. Algebra 319 (2008), no. 8, 3128-3140.

[2] H. E. Bell, Near-rings in which each element is a power of itself, Bull. Austral. Math. Soc. 2 (1970), 363-368.

[3] G. F. Birkenmeier, H. E. Heatherly, and E. K. Lee, Completely prime ideals and associated radicals, Ring theory (Granville, OH, 1992), 102-129, World Sci. Publ., River Edge, NJ, 1993.

[4] P. M. Cohn, Reversible rings, Bull. London Math. Soc. 31 (1999), no. 6, 641-648.

[5] J. L. Dorroh, Concerning adjunctions to algebras, Bull. Amer. Math. Soc. 38 (1932), no. $2,85-88$. 
[6] K. E. Eldridge, Orders for finite noncommutative rings with unity, Amer. Math. Monthly 75 (1968), no. 5, 512-514.

[7] S. U. Hwang, Y. C. Jeon, and Y. Lee, Structure and topological conditions of NI rings, J. Algebra 302 (2006), no. 1, 186-199.

[8] N. Jacobson, Some remarks on one-sided inverses, Proc. Amer. Math. Soc. 1 (1950), $352-355$.

[9] J. Y. Kim, Certain rings whose simple singular modules are GP-injective, Proc. Japan Acad. Ser. A Math. Sci. 81 (2005), no. 7, 125-128.

[10] J. Y. Kim and J. U. Baik, On idempotent reflexive rings, Kyungpook Math. J. 46 (2006), no. 4, 597-601.

[11] N. K. Kim, Y. Lee, and Y. Seo, Structure of idempotents in rings without identity, (submitted).

[12] T. K. Kwak and Y. Lee, Reflexive property of rings, Comm. Algebra, 40 (2012), no. 4, 1576-1594.

[13] T. K. Kwak, Y. Lee, and S. J. Yun, The Armendariz property on ideals, J. Algebra 354 (2012), 121-135.

[14] J. Lambek, Lectures on Rings and Modules, Blaisdell Publishing Company, Waltham, 1966.

[15] T. K. Lee and T. L. Wong, On Armendariz Rings, Houston J. Math. 29 (2003), no. 3, 583-593.

[16] T. K. Lee and Y. Q. Zhou, Armendariz and reduced rings, Comm. Algebra 32 (2004), no. 6, 2287-2299.

[17] G. Marks, On 2-primal Ore extensions, Comm. Algebra 29 (2001), no. 5, 2113-2123.

[18] G. Mason, Reflexive ideals, Comm. Algebra 9 (1981), no. 17, 1709-1724.

[19] J. C. McConnell and J. C. Robson, Noncommutative Noetherian Rings, John Wiley \& Sons Ltd., 1987.

[20] M. B. Rege and S. Chhawchharia, Armendariz rings, Proc. Japan Acad. Ser. A Math. Sci. 73 (1997), no. 1, 14-17.

[21] J. C. Shepherdson, Inverses and zero-divisors in matrix ring, Proc. London Math. Soc. (3) 1 (1951), 71-85.

[22] L. Xu and W. Xue, Structure of minimal non-commutative zero-insertive rings, Math. J. Okayama Univ. 40 (1998), 69-76.

TAI KeUn KWAK

Department of Mathematics

DAEJIN UNIVERSITY

Pocheon 487-711, KoreA

E-mail address: tkkwak@daejin.ac.kr

YANG LEE

Department of Mathematics Education

Pusan National University

Pusan 609-735, KoreA

E-mail address: ylee@pusan.ac.kr 